\title{
Removal of the Mixture of Azo Dyes Allura Red - Tartrazine by Adsorption onto Corncob
}

\author{
Sara Jiménez, Carolina Velásquez, Felipe Mejía, Angelina Hormaza \\ Universidad Nacional de Colombia, Faculty of Sciences \\ St. 59a \#63-20, Medellín, Colombia \\ sjimenezc@unal.edu.co; cvelasquezq@unal.edu.co; fmejiao@unal.edu.co; ahormaza@unal.edu.co
}

\begin{abstract}
Synthetic dyes are widely used around the world, and big volumes of coloured effluents end up being discharged into water bodies. Allura Red and Tartrazine are azo dyes with a broad use spectrum mainly in food and textile industries and their removal from the aquatic ecosystems, is a challenge. In the last decades, several methods have emerged to find a solution of this environmental problem, among them, adsorption using agro-industrial by-products stands out as a high efficient and low cost metodology for the removal of dyes, both individual and in mixture. Thus, the aim of this work was to confirm the reliability of spectrophotometric techniques for quantifying coloured compounds and then to evaluate the adsorption process of the mixture Allura Red - Tartrazine through a $2^{3}$ full factorial design with a central point. The Lambert-Beer law allows to quantify with errors lower than $2.0 \%$. The best percentage of adsorption of the azo dyes mixture, in order of $94.92 \%$, was achieved with a dosage of $20 \mathrm{~g} / \mathrm{L}$ and concentrations of 20 ppm for both dyes. These results show that adsorption using agricultural wastes is a promising alternative for treating mixtures of dyes in solution.
\end{abstract}

Keywords: Agro-industrial waste, Multicomponent Systems, Spectrophotometry, Statistical design, Synthetic dyes

\section{Introduction}

The depletion and decline of water resources worldwide is a concerning environmental issue. Synthetic dyes are one of the most common pollutants of water, because they are easy handling, and are highly resistant to light, temperature, detergents and microbial attack [1]. Several industries, such as pharmaceutic, textile and food, use these molecules during the dyeing procedures, and due to the inefficiency of these processes, large volumes of coloured effluents are generated and discharged into the aquatic ecosystems [2]. Particularly, the azo dyes Allura Red, AR, and Tartrazine, TA, are widely used in food industries in despite of their high toxicity $[3,4]$.

Seeking to solve the environmental problem of dyes discharge, different physical and chemical methodologies have been applied. There are many reports about the use of fenton reagent, ozonation, electrochemical destruction, adsorption onto activated carbon, electrokinetic coagulation, and others for dye degradation, in which high decoulorization percentages have been obtained. However, troubles associated with high costs, incomplete removal, breakdown of these pollutants and toxic by-product formation restrict their use [5]. Adsorption onto agro-industrial wastes has emerged as a viable alternative because of its low cost and positive environmental impact. Besides, these non-conventional adsorbents are available in a huge amount and they usually entail cumulation problems [6]. Moreover, agricultural wastes have proven great efficiencies in the removal of dissolved dyes, both individual and in mixture, with adsorption percentages up to $60 \%$ $[7,8]$.

There are a few studies related with adsorption of dye mixtures in comparison with those for single-component. One of this reason is probably the difficulty to quantify the analytes, methods such as chromatography[9], mass spectrometry [10] and capillary electrophoresis[11] usually require complex techniques, extended scanning times or expensive equipment. Although, spectrophotometric methods, based on Lambert-Beer law, are less reported, they represent a reliable and time-saving methodology when the single-components in the mixture do not react but their spectra are overlapped[12, 13].

This research aimed to confirm that the spectrophotometric method is an accurate technique in the quantification of dyes in mixture. Besides, the removal of the mixture AR-TA was evaluated by adsorption onto the agricultural waste 
corncob, CC, through a $2^{3}$ full factorial design, where the selected factors were the dosage of adsorbent, D, and the concentration of both dyes AR and TA.

\section{Materials and Methods}

\subsection{Adsorbent Pre-Treatment and Solution Preparation}

Corncob was acquired from the local market. It was washed, dried at $100^{\circ} \mathrm{C}$, ground in a microfine mill and then it was sieved. Particles ranging in size from 300 to $700 \mu \mathrm{m}$ were chosen for performing the adsorption experiments. Both AR and TA are anionic dyes, for this reason, the different solutions were prepared in distilled water at $\mathrm{pH} 2.0$. Additionally, concentrations in the range of 1.0 to $40.0 \mathrm{ppm}$ for both dyes were selected for the calibration curves.

\subsection{Quantification of the Mixture of Dyes}

As can be seen, in the absorption spectrum of the mixture AR - TA the peaks of maximum absorbance of each dye are overlapped, Fig. 1.

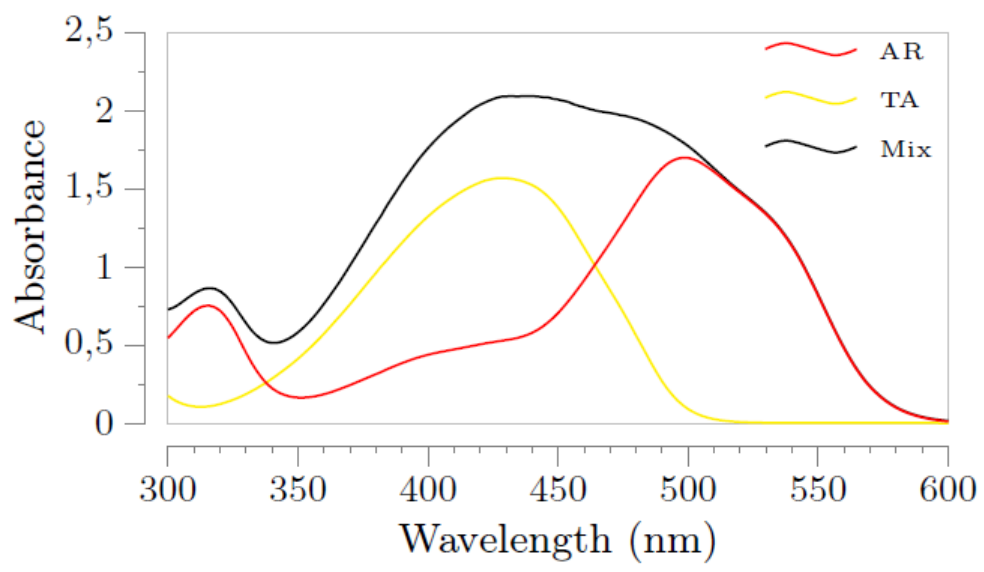

Fig. 1: Absorption spectrum of both, the mixture AR - TA and the single-components.

Due to the above, there are not wavelengths $(\lambda)$ in which each dye can be exclusively quantified. However, the absorbance is an additive property when the single components do not react. Then, from the Lambert Beer law the following equations are obtained:

$$
\begin{gathered}
A_{\lambda 1}=\varepsilon_{A R, 1} b C_{A R}+\varepsilon_{T A, 1} b C_{T A} \\
A_{\lambda 2}=\varepsilon_{A R, 2} b C_{A R}+\varepsilon_{T A, 2} b C_{T A}
\end{gathered}
$$

Where, $A_{\lambda 1}$ and $A_{\lambda 2}$ are the absorbances at $\lambda 1=500 \mathrm{~nm}$ and $\lambda 2=428 \mathrm{~nm}$, which are the maximum absorption wavelength of the AR and TA respectively. $\varepsilon_{A R, I}$ and $\varepsilon_{A R, 2}$ are the molar absorptivities of the AR at $\lambda 1$ and $\lambda 2, \varepsilon_{T A, I}$ and $\varepsilon_{T A, 2}$ are the molar absorptivities of the TA at $\lambda 1$ and $\lambda 2$, b is the optical path $(1.0 \mathrm{~cm})$ and $C_{A R}$ and $C_{T A}$ are the concentrations of AR and TA correspondingly. From Eqs. (1) - (2) more direct expressions for AR and TA concentrations in terms of the absorbances could be established, which were measured in a spectrophotometer Perkin Elmer UV-Vis Lambda 35.

$$
\begin{aligned}
C_{A R} & =\frac{\varepsilon_{T A, 2} A_{\lambda 1}-\varepsilon_{T A, 1} A_{\lambda 2}}{\varepsilon_{A R, 1} \varepsilon_{T A, 2}-\varepsilon_{A R, 2} \varepsilon_{T A, 1}} \\
C_{T A} & =\frac{\varepsilon_{A R, 1} A_{\lambda 2}-\varepsilon_{A R, 2} A_{\lambda 1}}{\varepsilon_{A R, 1} \varepsilon_{T A, 2}-\varepsilon_{A R, 2} \varepsilon_{T A, 1}}
\end{aligned}
$$




\subsection{Full Factorial Design}

The adsorbent dosage and the concentration of every component of the mixture were evaluated in a $2^{3}$ full factorial design with central point. The levels of each factor are described in table 1 . The statistical analysis was performed using the $\mathrm{R}$ software/environment[14]. At the time of this writing, R-3.2.3 was available and the package agricolae was used[15].

Table 1: Factors of the $2^{3}$ full factorial design.

\begin{tabular}{|c|c|c|c|}
\hline Factors / Levels & Low & Central & High \\
\hline $\mathrm{D}\left(g_{c c}=\mathrm{L}_{s l n}\right)$ & 10 & 15 & 20 \\
\hline$C_{A R}(\mathrm{ppm})$ & 20 & 30 & 40 \\
\hline$C_{T A}(\mathrm{ppm})$ & 20 & 30 & 40 \\
\hline
\end{tabular}

\section{Results and Discussion}

\subsection{Quantification of the Dye Mixture}

The behaviour of each dye, both individually and in mixture, allow to set the detection limits for performing the calibration curves. From these curves, the following molar absorption coefficients to quantify each dye were established:

$$
\varepsilon_{A R, I}=0.0426 \quad \varepsilon_{A R, 2}=0.0134 \quad \varepsilon_{T A, I}=0.0022 \quad \varepsilon_{T A, 2}=0.0394
$$

These constants have an average error of $0.84 \%$ and $1.96 \%$ in the quantification of AR and TA respectively. On the subject, Turabik (2008) reported errors of the same order for quantifying a mixture of basic dyes by first order derivative spectrophotometric analysis method [16]. Therefore, these results confirm that spectrophotometric methods are reliable for determining dye concentrations in binary systems.

\subsection{Full Factorial Design}

The different assays, as well as the global removal percentage are presented in table 2. According to the Scheffé test for multiple comparisons, means with the same letter are not significantly different with a confidence level of $95 \%$.

Table 2: Global removal percentage for each treatment.

\begin{tabular}{|c|c|c|c|c|}
\hline Assay & $\mathrm{D}\left(g_{c c}=L_{s l n}\right)$ & $C_{A R}(\mathrm{ppm})$ & $C_{T A}(\mathrm{ppm})$ & \% Adsorption \\
\hline 1 & 10 & 40 & 40 & $(57.69 \pm 10.85) \mathrm{c}$ \\
\hline 2 & 10 & 40 & 20 & $(74.18 \pm 3.80) \mathrm{abc}$ \\
\hline 3 & 10 & 20 & 40 & $(67.63 \pm 6.99) \mathrm{bc}$ \\
\hline 4 & 10 & 20 & 20 & $(86.09 \pm 4.90) \mathrm{ab}$ \\
\hline 5 & 15 & 30 & 30 & $(87.33 \pm 5.74) \mathrm{ab}$ \\
\hline 6 & 20 & 40 & 40 & $(86.04 \pm 8.77) \mathrm{ab}$ \\
\hline 7 & 20 & 40 & 20 & $(89.66 \pm 7.84) \mathrm{ab}$ \\
\hline 8 & 20 & 20 & 40 & $(87.13 \pm 6.40) \mathrm{ab}$ \\
\hline 9 & 20 & 20 & 20 & $(94.92 \pm 4.75) \mathrm{a}$ \\
\hline
\end{tabular}

The best percentage of adsorption, 94.92\%, was achieved with a dosage of $20 g_{c c}=L_{s l n}$ (high level) and concentrations of $20 \mathrm{ppm}$ for both dyes (low level), this result agree with the reported by Echavarria et al. (2014), who found that the adsorption increased when a greater amount of adsorbent is used[7]. This could be attributed to the increase in the adsorption sites on the corncob surface. Furthermore, low dye concentrations represent less competition between the molecules to access to active sites of the adsorbent, therefore the adsorption is more efficient. Despite, the groups do not show a definite trend among the factors, the treatments 1-3 (dosage $10 g_{c c}=L_{s l n}$ ) led to the lowest removals, probably due to the lower dosage. 
On the other hand, the analysis of variance table 3, shows that the first order interactions between D: $C_{A R}$ and D: $C_{T A}$ are significant with a $p$-value lower than 0.05 . Because of these reasons, it is not appropriated to evaluate the principal effect of the factors involved in each interaction. The simple outcome of every parameter is too different to have an average, it means, the effect on the response variable is not additive.

Table 3: Analysis of variance.

\begin{tabular}{|c|c|c|c|c|c|}
\hline & Df & Sum Sq. & Mean Sq. & F-value & $p$-value \\
\hline Block & 2 & 589.0 & 294.5 & 18.339 & 0.000123 \\
\hline $\mathrm{D}$ & 1 & 1952.4 & 1952.4 & 121.588 & 0.000000 \\
\hline$C_{A R}$ & 1 & 298.4 & 298.4 & 18.582 & 0.000718 \\
\hline$C_{T A}$ & 1 & 806.3 & 806.3 & 50.211 & 0.000005 \\
\hline $\mathrm{D}: C_{A R}$ & 1 & 90.1 & 90.1 & 5.608 & 0.032807 \\
\hline $\mathrm{D}: C_{T A}$ & 1 & 207.9 & 207.9 & 12.948 & 0.002908 \\
\hline$C_{A R}: C_{T A}$ & 1 & 14.1 & 14.1 & 0.881 & 0.363796 \\
\hline $\mathrm{D}: C_{A R}: C_{T A}$ & 1 & 1.8 & 1.8 & 0.112 & 0.742294 \\
\hline Residuals & 14 & 224.8 & 16.1 & & \\
\hline
\end{tabular}

\section{Conclusion}

The results obtained in this study confirm that the spectrophotometric method is an accurate and reliable technique for quantifying dyes in binary systems, with errors lower than $2.0 \%$. Besides, the adsorption assays indicated that a greater dose of adsorbent enhances the removal of dyes from effluents, and supported the high potential of corncob as a nonconventional, low-cost and easily available adsorbent for removal of the mixture of dyes AR-TA. Furthermore, these findings promote research on the adsorption of other multicomponent systems and their scale-up.

\section{Acknowledgments}

The authors thank Universidad Nacional de Colombia Sede Medellín for its support through the infrastructure of the Experimental Chemistry Laboratory as well as the funding of COLCIENCIAS through the call 745, Project Code 111871250685.

\section{References}

[1] C. ONeill, F. R. Hawkes, D. L. Hawkes, N. D. Loureno, H. M. Pinheiro, and W. Dele, "Colour in textile effluents sources, measurement, discharge consents and simulation: a review," Journal of Chemical Technology and Biotechnology, vol. 74, no. 11, pp. 1009-1018, 1999.

[2] E. Forgacs, T. Cserhti, and G. Oros, "Removal of synthetic dyes from wastewaters: A review," Environ. Int., vol. 30, pp. 953-971.

[3] Anonymous, "Allura red- developmental and psychotoxic effects?" Food Chem. Toxicol., vol. 22, no. 11, pp. 913928, 1984.

[4] H. Ashida, T. Hashimoto, S. Tsuji, K. Kanazawa, and G. Dannoi, "Synergistic effects of food colors on the toxicity of 3-amino-1, 4-dimethyl -5 h-pyrido [4, 3-b] indole (trp-p-1) in primary cultured rat hepatocytes," Nutr. Sci. Vitaminol, vol. 46, pp. 130-136, 2000.

[5] T. Robinson, G. Mcmullan, R. Marchant, and P. Nigam, "Remediation of dyes in textile effluent: a critical review on current treatment technologies with a proposed alternative," Bioresour. Technol., vol. 77, pp. 247-255, 2001.

[6] G. Crini, "Non-conventional low-cost adsorbents for dye removal: A review," Bioresour. Technol., vol. 97, no. 9, pp. 1061-1085, 2006.

[7] A. M. Echavarria-Alvarez and A. Hormaza-Anaguano, "Flower wastes as a low-cost adsorbent for the removal of acid blue 9," Dyna, vol. 81, no. 185, pp. 131-137, 2014.

[8] V. K. Gupta, S. Khamparia, I. Tyagi, D. Jaspal, and A. Malviya, "Decolorization of mixture of dyes: A critical review," Glob. J. Environ. Sci. Manag., vol. 1, no. 1, pp. 71-94, 2015. 
[9] R. A. Hoodless, K. G. Pitman, T. E. Stewart, J. Thomson, and J. E. Arnold, "Separation and identification of food colours," J. Chromatogr., vol. 54, pp. 393-404, 1971.

[10] K. Harada, K. Masuda, and M. Suzukit, "Separation and identification of food dyes by thin-layer chromatography/ liquid secondary ion mass spectrometry," Biol. Mass Spectrom., vol. 20, pp. 522-528, 1991.

[11] S. Suzuki, M. Shirao, M. Aizawa, H. Nakazawa, K. Sasa, and H. Sasagawa, "Determination of synthetic food dyes by capillary electrophoresis," J. Chromatogr. A, vol. 680, no. 2, pp. 541-547, 1994.

[12] G. Mckay and B. al Duri, "Prediction of multicomponent adsorption equilibrium data using empirical correlations," Chem. Eng. J., vol. 41, pp. 9-23, 1989.

[13] A. Kurniawan, H. Sutiono, N. Indraswati, and S. Ismadji, "Removal of basic dyes in binary system by adsorption using rarasaponin-bentonite: Revisited of extended langmuir model," Chem. Eng. J., vol. 189-190, pp. 264-274, 2012.

[14] R: A language and environment for statistical computing. R Foundation for Statistical Computing. [Online]. Available: https://cran.r-project.org/

[15] Agricolae: Statistical procedures for agricultural research. [Online]. Available: https://cran.r-project.org/

[16] M. Turabik, "Adsorption of basic dyes from single and binary component systems onto bentonite: Simultaneous analysis of basic red 46 and basic yellow 28 by first order derivative spectrophotometric analysis method," $J$. Hazard. Mater., vol. 158, pp. 52-64, 2008. 